\title{
Enabling Exclusive Shared Access to Cloud of Things Resources
}

\author{
Ahmed Salim Al Rawahi, Kevin Lee, Jon Robinson, Ahmad Lotfi \\ ahmed.alrawahi2016@my.ntu.ac.uk \\ kevin.lee, jon.robinson, ahmad.lotfi\}@ntu.ac.uk
}

\begin{abstract}
Cloud of Things (CoT) is an emerging paradigm that integrates Cloud Computing and Internet of Things (IoT). CoT is constrained by the limited computing capabilities of IoT resources and the costly investment required to deploy IoT infrastructure. Despite the support of existing CoT implementations to various applications, IoT physical resources are still computationally limited and cannot to be shared as other Cloud resources yet. This paper proposes a new approach to improve shared access to IoT resources. The new approach relies on optimising resource trading of IoT resources to enable exclusive access to allocated resources at a given time. A generic architecture is proposed to support the proposed approach along with notations required to commoditise IoT resources. A case study of multiple application uses is presented. Simulations are carried out to evaluate the feasibility of the approach using three optimisation techniques. The evaluation of the proposed approach includes optimising the cost of resource allocation, different QoS metrics and the coverage of IoT resources.
\end{abstract}

Keywords: Cloud of Things, Internet of Things, Shared Access, Optimisation

\section{Introduction}

IoT is increasingly attracting the attention of public, industry and academia due to its ability in monitoring and responding to many real-world events. Existing applications are constrained by considerable financial investments needed to deploy IoT infrastructures and by the limited computing capabilities of IoT resources. These constraints limit the attained benefits of IoT.

Cloud Computing is viewed as a complementary technology to overcome the existing constraints of IoT. Cloud applications do not usually require any costly investment in physical infrastructure and can provide virtually unlimited computing resources. Considerable efforts from academia and industry are being made to integrate Cloud Computing and IoT into a new paradigm in response to the requirements of emerging applications. The new paradigm is commonly called Cloud of Things (CoT). 
As in other large-scale computing infrastructures, resource management is becoming a challenging and complex task in CoT. The complexity resides here for two reasons. The first is due to the heterogeneity of IoT resources which is difficult to quantify their value and leading to the involvement of multifaceted variables and decisions. The second is due to the constrained nature of IoT resources in terms of computing capabilities which is challenging to enable an efficient sharing mechanism to the IoT physical resources.

This paper introduces the concept of exclusive shared access (ESA) to CoT resources. The proposed approach uses market-based mechanisms to quantify the value of heterogeneous IoT resources and commoditise them. Optimisation strategies can be employed to map requests to the optimal Io $T$ resources that can satisfy the requests. Then, exclusive access to matched resources at a given time can be scheduled. This approach is a key enabler for sharing the constrained IoT physical resources with considerable flexibility.

This approach is justified as follows. Market-based mechanisms have been used to quantify and commoditise resources in similar large-scale computing infrastructure including Grids, Cloud Computing and Wireless Sensor Networks (WSNs) [1]. Optimisation techniques are well known for their capabilities in finding optimal solutions to similar complex problems in various domains.

The remainder of this paper is as follows. Section 2 reviews the related work. Section 3 introduces the proposed approach, defines the problem and describes the system architecture. A case study with multiple application scenarios is discussed in Section 4 . Section 5 presents experimental evaluation and results. Section 6 draws the conclusions.

\section{Background}

Resource allocation tasks in large-scale computing systems are often described as NP-hard. NP-hard problems have no best or exact solutions in a given time due to their complexity. CoT is a large-scale computing infrastructure by nature and its resource allocation is challenging. The proposed approach is inspired by a research direction that employs trading mechanisms to allocate resources in similar large-scale computing environments to CoT. This section discusses this research trend.

Multiple models and architectures have been proposed in [2-5] to create a market-based value for IoT resources. Despite their diversity in aims and techniques, the problem is commonly defined as an optimisation problem. The architecture proposed in [2] used a Cloud broker to trade IoT resources using different optimisation strategies. The optimisation is used to minimise response time, system energy consumption and to maximise the broker's profit. A model to support CoT resource providers has been proposed in [3] where the optimisation is used to maximise resource utilisation.

Another direction has been taken by [6] to virtualise and allocate IoT resources. The optimisation is applied to match IoT virtual objects to application requests with some quality constraints. Another Quality of service 
model for IoT resource allocation has been developed in [7]. The optimisation aims to improve scheduling performance of IoT network and reduce the resource costs while maintaining the QoS constraints of information accuracy, energy consumption and resource coverage. The optimisation is also applied in [8] to schedule IoT applications on a multi-Cloud system aiming to improve the system performance and reduce the cost.

Existing techniques of sharing Cloud-based IoT resources can be categorised into 1) service-oriented approaches and 2) software-oriented approaches. Service-oriented approaches are mainly focused on sharing data and/or the virtualised IoT resources as services $[9,10]$. The shared data is sent from various distributed IoT resources to a back-end Cloud for further processing by multiple users. Similarly, virtualised IoT services are built on the top of physical IoT resources as Cloud services where multiple users utilise the virtualised resources but not the physical ones. Software-oriented approaches focus on enabling sharing IoT resources by enabling multiple application access to IoT devices using middleware [11,12].

Limitations of service-oriented approaches include IoT physical resources are not actually shared and the virtualisation techniques may accelerate depletion rate of battery powered resources that result in minimising the lifetime of resources. Software-oriented approaches are still emerging and subject to improve heterogeneity, scalability and dynamism aspects of IoT. Optimisation is also not considered to improve either technical aspects or market-based mechanisms. Such limitations provide valuable perceptions for the proposed solution to fill that gap. This paper builds upon [4] to introduce the concept of exclusive shared access (ESA) to CoT resources and to evaluate the use of optimisation strategies when implementing the proposed approach in trading CoT resources.

\section{Exclusive Shared Access to CoT Resources}

Resource sharing mechanisms in Cloud Computing matured over time while approaches to sharing IoT resources are still emerging. One of the major differences between the two types of resources is their capabilities. Cloud resources are usually hosted in powerful large-scale data-centres to provide virtually unlimited, elastic and on-demand computing resources. Conversely, IoT resources are widely distributed across the application area with constrained computational and power resources.

\subsection{The Proposed Approach}

The proposed solution in this paper is described as follows. A marketplace system receives requests from consumers and resources from providers. Marketbased notations are used to quantify the value of IoT physical resources and the requests. Based on the goal of the marketplace, an optimisation strategy is used to perform two tasks as follows. 1) Map the requests to resources that satisfy 
them. 2) Evaluate the mapped assignments of requests and resources to propose an optimal assignment. The optimal assignment is scheduled as presented in the following sections.

The concept of exclusive shared access describes the process of scheduling IoT physical resources to be accessed and utilised by a single consumer at a given time and by multiple consumers at the length of the schedule. The concept is two fold. 1) Exclusive access by each consumer to the desired resources at the required time. 2) Shared access for multiple consumers to the same resources throughout the schedule. When the utilisation time of a consumer elapses, the resources are released and assigned to the next consumer in the schedule. When the schedule completes, the assigned resources are totally released back to the proposed system for a new round of assignment to different consumers.

To the best of the authors' knowledge, this work is the first to coin the concept of Exclusive Shared Access (ESA) to CoT resources. It is also the first to implement the concept in trading CoT setup using different optimisation strategies.

\subsection{ESACoT Marketplace System Architecture}

The proposed approach relies on commoditising CoT resources to enable efficient sharing. A generic marketplace architecture is proposed in Fig. 1 to support the implementation of the concept. The proposed marketplace and the trading process is described as follows.

The marketplace can be composed of minimum two components. The optimisation tool receives requests from consumers and resources from the providers. The tool map requests to all potential resources. Based on the market goal, the evaluation of each proposed map is performed. This may include 1) Cost-based objectives (e.g. consumer's cost, provider's profit) 2) Time-based objectives (e.g. latency between consumers and providers) 3) Performance-based objectives (e.g Coverage of IoT node). The second task of the optimisation tool is to evaluate a large number of mapped resources and requests. The evaluation ranks each map based on the objective and its compliance with search constraints discarding maps that either extreme (e.g very expensive resource) or violate the constraints (e.g below certain energy level).

Upon finding the optimal map, the optimisation tool submits it to the scheduler to start scheduling and allocating the resources. The scheduler maintains a schedule for all resources and requests in the optimal map. The scheduler manages the lease-time of each consumer to join and dis-join resources accordingly. Each resource has an exclusive access from a consumer at a given time. When the lease time of the consumer elapses, the resource is released and a different consumer is granted the exclusive access. As it is shown in Fig. 2, each resource can be consumed by multiple applications at the length of the schedule. 


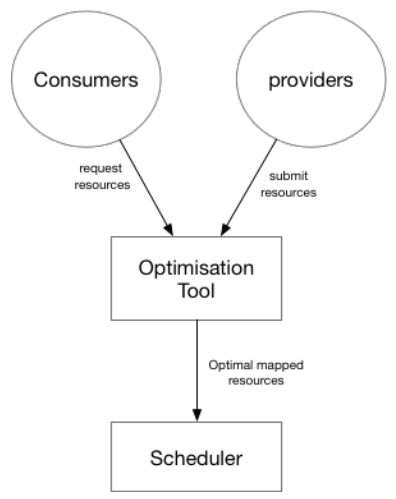

Figure 1: High-level CoT Marketplace Architecture

This architecture is designed with consideration of flexibility and dynamism required in CoT. The optimisation tool requires minimal changes either by using a different objective or a different optimisation strategy. The approach reduces the time needed to find an optimal map for real-time IoT applications. The advantages of this approach include the following.

1. Improving Interoperability: IoT physical resources are truly utilised when consumers are not restricted to specific infrastructure and can move their applications to different providers due to changes in requirements or market offerings. The proposed approach is implemented by a marketplace where heterogeneous vendor-independent and platform-independent resources can be utilised by various IoT applications.

2. Reducing Costs: It is a cost-effective approach that separates between IoT application development and IoT infrastructure deployment. Infrastructure deployers can deploy their IoT resources independently without considering application-specific requirements. Similarly, application developers can develop their applications without usual concerns about infrastructure complexity and costs. The cost is reduced for application developers as they do not require a dedicated IoT infrastructure and any maintenance or specialised personnel to deploy it. Infrastructure owners reduce their application development costs and increase their revenue from the trading which may justify the return on investment of IoT infrastructure that can be very costly and infeasible for many applications. This will likely reduce the overall costs and motivate new services and application.

3. Providing Flexibility: The proposed approach provides a significant flexibility to various IoT applications. For instance, time-sensitive applications including law enforcement and emergency agencies can gain high priority access to various IoT resources to monitor and respond to 
incidents as needed in real-time. More case uses are described in Section 4 .

\subsection{Problem Statement}

The marketplace system $\mathrm{M}$ receives $\mathrm{n}$ number of requests $\mathrm{RQ}=\left(r q_{1}, \ldots, r q_{i}\right)$ and m number of resources $\mathrm{R}=\left(r_{1}, \ldots, r_{j}\right)$. Providers submit their resources and consumers submit their requests to the marketplace where the optimisation tool aims first to map the requests to the suitable resources and then evaluate the maps to find an optimal solution that satisfies an objective. The evaluation of the mapped assignments is performed upon quantified resource attributes RA= $\left(r a_{1}, \ldots, r_{k}\right)$. Attributes describes IoT node properties including but not limited to processing, memory, sensing, storage and energy capabilities of the node. In CoT environment, many decision variables can be considered for optimisation. In this section, notations required for trading CoT resources are defined in table 1.

Trading of $\mathrm{CoT}$ resources is formulated as a bin-packing optimisation problem where different optimisation algorithms are applied including Non-dominated Sorting Genetic Algorithm II (NSGA2) [13], Covariance Matrix Adaptation Evolution Strategy (CMA-ES ) [14] and The Third Evolution Step of Generalized Differential Evolution Algorithm (GDE3) [15]. The objectives considered in this paper are described as follows.

Objective 1: Minimising consumer's cost. The consumers always aim to minimise their costs. To achieve this objective, the resource cost is considered for minimisation. The cost of the resource is set to $c s_{j}$ while the consumer bid is donated by $b_{i}$. Requests from consumers specify a utilisation time donated by $t_{i}$. Transmission and delay time is also considered and set to $T Q_{i j}$. It can be measured as $T Q_{i j}=T_{i j} \div d l_{i j}$ where $T_{i j}$ is the latency between consumer $i$ and provider $j$ while $d l_{i j}$ is the distance between a requested resource from consumer $i$ and the actual location of the resource from provider $j$. The objective of minimising the consumer cost is presented as follows.

$$
\begin{gathered}
\text { Minimise } \quad c o s t=\left\{\left(b_{i}-c s_{j}\right) \times\left(t_{i}+T Q_{i j}\right)\right. \\
: i=1, \ldots, n ; j=1, \ldots, m\}
\end{gathered}
$$


Table 1: List of vocabularies

\begin{tabular}{cc}
\hline Vocabulary & Description \\
\hline $\mathrm{M}$ & The marketplace \\
$\mathrm{n}$ & Number of requests \\
$\mathrm{m}$ & Number of resources \\
$\mathrm{r}$ & Resource \\
$\mathrm{ra}$ & Resource attribute \\
$\mathrm{RA}$ & Set of resource attributes \\
$\mathrm{c}$ & Resource consumer \\
$\mathrm{p}$ & Resource provider \\
$b_{i}$ & Bid from consumer \\
$c s_{j}$ & Cost of a resource \\
$t_{i}$ & Lease time of requested resource \\
$r q_{i}$ & Request from consumer \\
$E_{j}$ & Energy consumption of a resource \\
$E r_{i}$ & Energy required by consumer \\
$E p_{j}$ & initial power supply of a resource \\
$E t_{\text {max }}$ & Maximum transmission power of resource $\mathrm{j}$ \\
$l_{j}$ & Location of a resource (latitude, longitude) \\
$d l_{i j}$ & Distance between a requested \\
$T_{i j}$ & resource and actual location of the resource \\
$t_{s t a r t}$ & Latency between consumer and provider \\
$t_{a c k}$ & Time of requesting a resource from a provider \\
$T Q i j$ & Time of receiving acknowledgement from a consumer \\
$s_{j}$ & Estimated queuing and transmitting delays \\
$C v_{j}$ & Sensing range of a resource \\
$c p_{i}$ & Area coverage of a resource \\
$c p_{j}$ & Number of requests from a consumer \\
$s e_{j}$ & Total Capacity of provider \\
\hline & Security features of a resource \\
\hline &
\end{tabular}




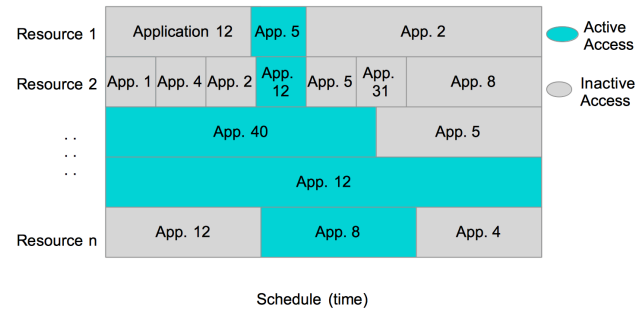

Figure 2: Schedule of Mapped Resources

Each provider has a limit of resources to provide. This is formulated as a capacity constraint in (2a) where $c p_{i}$ donates the number of requests from consumer $i$ and the capacity of a provider $j$ is set to $c p_{j}$. Constraint (2b) ensures both the cost of a resource and the bid from a consumer are always positive. A bid $b_{i}$ from consumer $i$ has to be greater than the initial cost of the resource $c s_{j}$.

Energy constraint in (2c) limits the required energy $E r_{i}$ to the resource energy $E p_{j}$. Constraint (2e) ensures that the resource security features $s e_{j}$ is better than or equal the required ones $s e_{i}$. The coverage constraint (2f) is to ensure the resource coverage $C v_{j}$ over the distance $d l_{i j}$ between requested location and the actual location of the resource.

Objective 2: Maximising The Resource Coverage. Resource coverage is likely to be an important objective for many IoT consumers. The coverage of IoT resources can be measured by considering the sensing range $s_{j}$ and the maximum transmission power $E t_{\max }$ of a resource. The area coverage of a resource $C v_{j}$ and the distance $d l_{i j}$ between requested location and the actual location of the resource are also considered. To evaluate the resource coverage, the following objective is formulated.

$$
\begin{array}{r}
\text { Maximise } \quad C v=\left\{\frac{s_{j} \times E t_{\max }}{C v_{j}-d l_{i j}}: i=1,2, \ldots, n ; j=1,2, \ldots, m\right\} \\
\text { subject to }(2 a),(2 b),(2 d),(2 e),(2 f)
\end{array}
$$

The aim of the objective is maximise the coverage of the resources required by consumers. The same constraints introduced earlier apply.

\section{Case Study}

In this section, the following case study is discussed. The area around a high-traffic street of a metropolitan city is considered a desirable location for multiple enterprises and public organisations to implement their IoT applications. To elaborate, the following sub-sections maps resource providers and resource consumers to the architecture presented in Figure 1 and the application illustrated in Figure 3. 


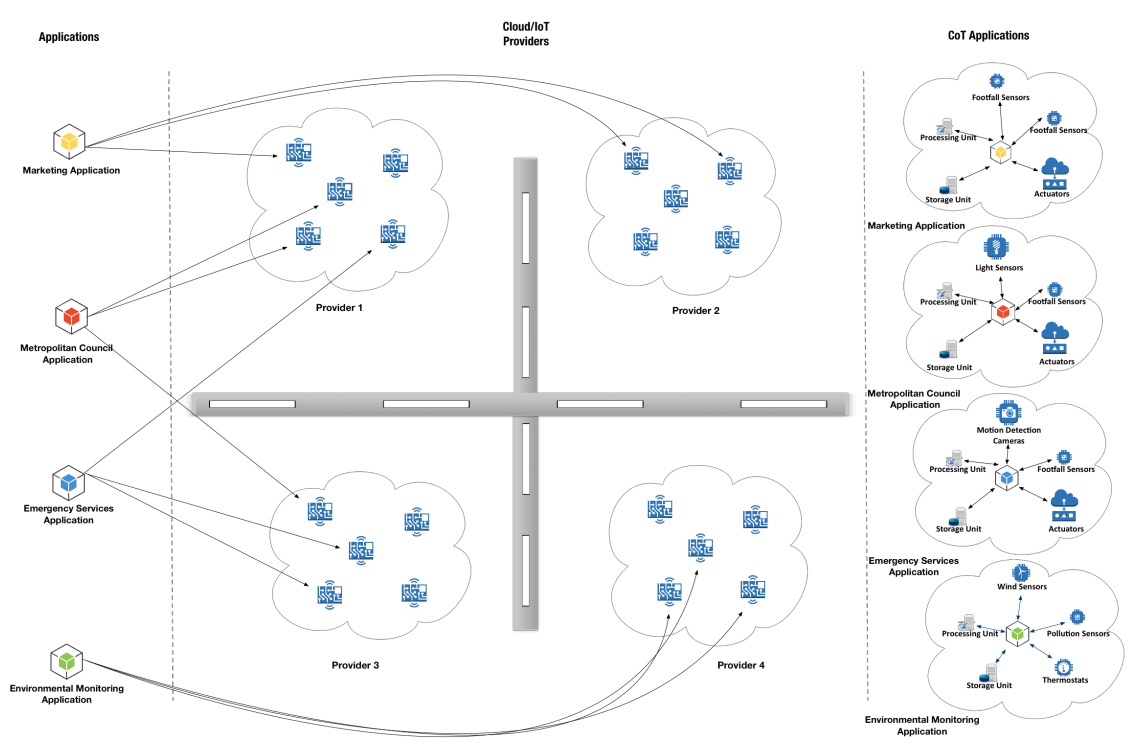

Figure 3: CoT Application

\subsection{Resource Providers}

In this case study, four providers deploy their networks of IoT resources across the considered area. Each network of a provider consists of multiple homogeneous nodes. Nodes of all providers become heterogeneous when compared with each others'. Each node consists of constrained computing capabilities that may differ from one to another. This may include a microprocessor, memory, a power supply, storage, sensor, actuator and network chip. IoT nodes are connected via their providers' area-wide wireless networks. The usage of different node types is discussed in the following section.

\subsection{Resource Consumers}

IoT resources can be consumed by a wide range of applications. Upon successful allocation of required resources, a consumer can send a software component (e.g Java applet or Python script) to configure and utilise the acquired resources based on the application requirements. In this case study, four applications are considered as IoT resource consumers including one business and three public organisations. The four presented applications support the vision of a smart city.

Marketing Application. A marketing agency owns electronic billboards around the area wants to develop an advertising application that uses statistics of pedestrians footfall across sidewalks. The agency can use footfall statistics along with other data sources to dynamically tailor selling of the electronic billboard spaces to clients. In this case, the agency would request a resource 
bundle of multiple footfall sensors, specifying a location (e.g 400meter $\times 10$ meter), undefined node processing power, constant energy source, undefined storage capacity, and network access, and certain security level.

Metropolitan Council Application. A metropolitan council has increasing responsibilities towards the metropolitan area of the city. The council plans to build an IoT application that can help making better-informed decisions. Pedestrian footfall is a good indicator of human activities within the area. It can be used to plan maintenance of sidewalks and pavements as well as building new ones. Maintenance projects within the area may require an installation of temporary traffic lights to control pedestrian activities and car traffic. Footfall sensors, traffic sensors and actuators play an important role in optimising the traffic within the maintained area especially during peak times. The sensors can measure pedestrian activities and density of the traffic while the actuators take control of traffic lights based on sensors readings. Light sensors can also be used to switch on/off street and sidewalks lights at the right time avoiding earlier or late switch on/off.

For long-term planning, the request would be for a bundle of any footfall sensors within the area, minimal storage and processing capacities, minimal network connectivity and basic security features. For day-to-day tasks, the request would be for a bundle of good light sensors, footfall sensors and actuators within 500meter $\times 500$ meter area. The power of the resources should be consistent, with adequate storage and processing units, responsive network access and good security characteristics.

Emergency Services Application. Metropolitan emergency services including police, ambulance and fire brigade want to build an IoT application that helps their teams accelerate their response to incidents. For instance, footfall can be used for crowd tracking and analysis during public events. It also allows to plan and aid evacuation procedures during incidents. Motion detection can be employed to early discover breaches of controlled zones. Using this application, emergency services can gain high priority access to a bundle of resources for short periods of time. For planning and prediction, the resource request would be for footfall sensors and motion detection cameras in a general location, with limited power, network, access and security characteristics. For a live emergency event, the request would be for the maximum number of resources around the incident location with the maximum reliability possible.

Environmental Monitoring Application. An environment agency aims to build an application for environmental impact analysis. The application is useful for monitoring and analysing various environmental indicators (e.g pollution, temperature, pressure, wind). These indicators help public decision-makers to control pollutions and promote environment-friendly lifestyles in the metropolitan area. The agency would request a bundle of distributed environmental sensors across the area. Footfall sensors can also help to gain a detailed picture of the environmental impact of activities in the area. As these applications are usually financially constrained, the bundle request would be submitted with minimal resources properties at the lowest 
price possible.

\section{Evaluation}

This section presents the simulations setup for Exclusive Shared Access Enabled CoT system. The results of evaluating different optimisation approaches are then analysed.

\subsection{Experimental Setup}

The simulated system is assumed to have 100 consumers with 100 requests each and 200 providers offering 200 resource each. The locations of all resources are randomly generated within 100 meter radius of a busy street in the city centre of Nottingham, UK. The locations are exact Latitude and Longitude $\left(x_{j}, y_{j}\right)$. It is assumed that each consumer requests homogeneous resources while each provider offer heterogeneous resources. The total number of requests is 10000 whereas the total number of resources is 40000 . The system uses the three optimisation strategies mentioned earlier to minimise the consumer cost and maximise the coverage of the resources. The three techniques implemented without modification or improvement using Python programming language. Both objective functions are evaluated individually as a single objective function. Simulations are configured up to 250 iterations and population size of 50. Simulations are performed in a computer with the following hardware specifications: Processor: $2.6 \mathrm{GHz}$ Intel Core i7, Memory: 16 GB $1600 \mathrm{MHz}$ DDR3.

\subsection{Experimental Results}

This section discusses the simulation results obtained. Figure 4 and Figure 5 show the best results of each objective function at specific iterations. The results show that CMS-ES contributes to the optimality of consumer cost and the resource coverage better than NSGA2 and GDE3. Despite the NSGA2 complexity, it converges faster than CMA-ES but falls into the local optima in both scenarios. This can be improved by using different parameters and operators. GDE3 also requires further parameters improvement as it is the lowest contributor in both scenario.

The results assert the feasibility of the proposed approach by using various optimisation strategies as a market mechanism for trading and sharing access to CoT resources. The proposed architecture demonstrates the flexibility and scalability of the approach in optimising objectives for that require mapping of a large number of requests and resources. The use of objective functions along with proposed notations shows their flexibility and effectiveness in quantifying the value of heterogeneous CoT resources.

Simulations limitations are summarised as follows. 1) Working with optimisation approaches may require trying different values of parameters (e.g. 


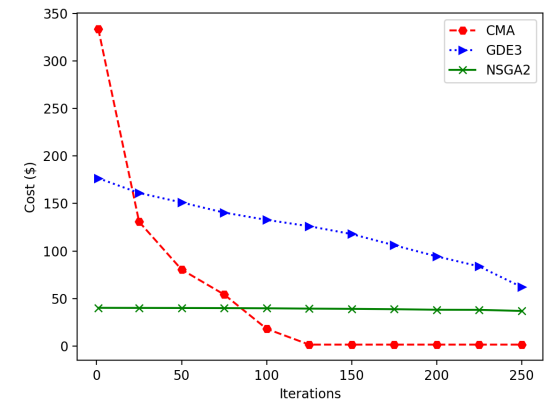

Figure 4: Minimisation of consumer cost

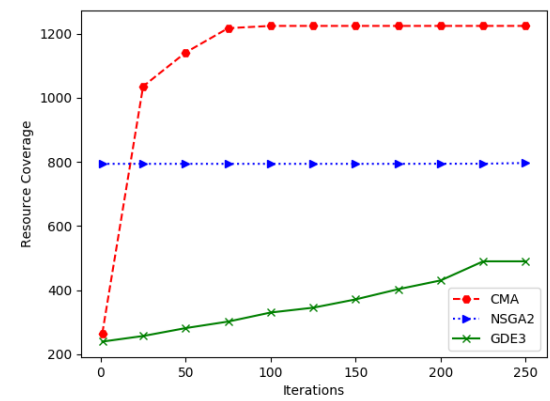

Figure 5: Maximisation of resource coverage

iteration, population size, mutation rate) to obtain satisfactory results. This can be computationally expensive and time-consuming. 2) Falling into the local optima (minima or maxima) may not be preventable in some scenarios by all optimisation techniques used in this paper.

\section{Conclusions and Future Work}

This paper introduces ESACoT, a new approach for sharing access to CoT resources. ESACoT minimises the costs of IoT application development as well as the deployment of IoT infrastructure. It maximises the interoperability between heterogeneous IoT resources and the flexibility required by various IoT applications.

Trading CoT resources using market-based optimisation techniques is the approach used to enable shared access to CoT resources. The objectives of consumer cost and the coverage of resources are optimised. The proposed architecture decreases the architectural complexity of CoT while maintains a high level of scalability by optimising objectives for a larger number of 
candidate solutions. Simulation results validate the feasibility of the approach in enabling shared access to constrained IoT resources.

Planned future work includes 1) Improving the mapping model for resources and requests 2) Developing the proposed architecture to address scalability, security and dynamism 3) Enhance the optimisation model to find better optimal solutions.

\section{References}

[1] K. Lee, G. Buss, and D. Veit, "A heuristic approach for the allocation of resources in large-scale computing infrastructures," Concurrency and Computation: Practice and Experience, vol. 28, no. 5, pp. 1527-1547, 2016.

[2] T. Kumrai, K. Ota, M. Dong, J. Kishigami, and D. K. Sung, "Multiobjective optimization in cloud brokering systems for connected internet of things," IEEE Internet of Things Journal, vol. 4, no. 2, pp. 404-413, 2017.

[3] I. Farris, L. Militano, M. Nitti, L. Atzori, and A. Iera, "Mifaas: A mobile-iot-federation-as-a-service model for dynamic cooperation of iot cloud providers," Future Generation Computer Systems, vol. 70, pp. 126137, 2017.

[4] A. S. Alrawahi, K. Lee, and A. Lotfi, "Trading of cloud of things resources," in Proceedings of the Second International Conference on Internet of Things and Cloud Computing, ser. ICC '17. New York, NY, USA: ACM, 2017, pp. 163:1-163:7. [Online]. Available: http://doi.acm.org/10.1145/3018896.3056780

[5] C. Perera and A. Zaslavsky, "Improve the sustainability of internet of things through trading-based value creation," in Internet of Things (WF-IoT), 2014 IEEE World Forum on. IEEE, 2014, pp. 135-140.

[6] V. Pilloni and L. Atzori, "Consensus-based resource allocation among objects in the internet of things," Annals of Telecommunications, vol. 72, no. 7-8, pp. 415-429, 2017.

[7] L. Li, S. Li, and S. Zhao, "Qos-aware scheduling of services-oriented internet of things," IEEE Transactions on Industrial Informatics, vol. 10, no. 2, pp. 1497-1505, 2014.

[8] I. A. Moschakis and H. D. Karatza, "Towards scheduling for internetof-things applications on clouds: a simulated annealing approach," Concurrency and Computation: Practice and Experience, vol. 27, no. 8, pp. 1886-1899, 2015.

[9] S. Abdelwahab, B. Hamdaoui, M. Guizani, and T. Znati, "Cloud of things for sensing-as-a-service: Architecture, algorithms, and use case," IEEE Internet of Things Journal, vol. 3, no. 6, pp. 1099-1112, 2016. 
[10] W. Li, I. Santos, F. C. Delicato, P. F. Pires, L. Pirmez, W. Wei, H. Song, A. Zomaya, and S. Khan, "System modelling and performance evaluation of a three-tier cloud of things," Future Generation Computer Systems, vol. 70, pp. 104-125, 2017.

[11] S. Akkermans, W. Daniels, G. Sankar R, B. Crispo, and D. Hughes, "Cerberos: a resource-secure os for sharing iot devices," in Proceedings of the 2017 International Conference on Embedded Wireless Systems and Networks. Junction Publishing, 2017, pp. 96-107.

[12] N. Small, S. Akkermans, W. Joosen, and D. Hughes, "Niflheim: An end-to-end middleware for applications on a multi-tier iot infrastructure," in Network Computing and Applications (NCA), 2017 IEEE 16th International Symposium on. IEEE, 2017, pp. 1-8.

[13] K. Deb, A. Pratap, S. Agarwal, and T. Meyarivan, "A fast and elitist multiobjective genetic algorithm: Nsga-ii," IEEE transactions on evolutionary computation, vol. 6, no. 2, pp. 182-197, 2002.

[14] N. Hansen, S. D. Müller, and P. Koumoutsakos, "Reducing the time complexity of the derandomized evolution strategy with covariance matrix adaptation (cma-es)," Evolutionary computation, vol. 11, no. 1, pp. 1-18, 2003.

[15] S. Kukkonen and J. Lampinen, "Gde3: The third evolution step of generalized differential evolution," in Evolutionary Computation, 2005. The 2005 IEEE Congress on, vol. 1. IEEE, 2005, pp. 443-450. 